\title{
Creating partnerships and improving health care: The role of genetic advocacy groups
}

Jannine D. Cody, $P h D^{1,2,3}$

Twenty-one years ago, our pediatrician called to ask me to stop by his office to pick up some laboratory results. My daughter Elizabeth had been born 6 weeks earlier with a severe bilateral cleft lip/palate and metatarsus adductus. These findings prompted closer scrutiny, and in our postpartum week in the hospital, many consultants were called in and tests were ordered. The fact that 6 weeks later the pediatrician was calling to tell me that results were in and ready to be picked up was not a surprise. Because Elizabeth's development seemed pretty normal, I was expecting to get a pile of normal results that would need to be filed in her rapidly expanding medical chart. I was not prepared for the news he was about to deliver.

He sat me down in his office, handed me a book, and said, "She has this." What she had was a missing piece from the end of the long arm of chromosome 18 (18q-). The book showed contorted unhappy babies and contained the following passage, "They are probably the most seriously afflicted among carriers of chromosome anomalies. They maintain the froglike position observed in infants and are reduced to an entirely vegetative and bedridden life."

The book also contained a long list of the other potential medical problems found in the 65 other individuals in the world with this condition. We spent the several next years and hundreds of doctor visits trying to figure out what on that list applied to our daughter. Even the obvious problems were not so straightforward. After 9 months of serial casting to correct her metatarsus adductus, her feet could be held in a neutral position but bounced back to their crescent shape as soon as the casts came off. The surgeries to repair her lip and palate kept coming apart and had to be re-repaired. We found out after her fifth surgery that she was growth hormone deficient, which can, and obviously did, result in healing problems. After numerous ear infections, a computed tomography scan revealed that she was actually having sinus infections. This meant we had not been treating her infections properly, and she had probably been suffering with a sinus infection for many months. Treatment of her sinus infection also resulted in eliminating her seemingly random inconsolable crying tantrums. When she was 3 years old she verbalized a lot, but had little

From the ${ }^{1}$ The Genetic Alliance; ${ }^{2}$ The Chromosome 18 Registry and Research Society; and the ${ }^{3}$ Department of Pediatrics, University of Texas Health Science Center at San Antonio, Texas. Jannine D. Cody, PhD, Department of Pediatrics, 7703 Floyd Curl Drive, San Antonio, TX 78229.

Submitted for publication August 13, 2006.

Accepted for publication August 29, 2006.

DOI: 10.1097/01.gim.0000250203.60283.10 understandable speech. Although she had passed numerous behavioral audiometry sessions, we discovered at age 3 years that she was hearing impaired, which at that age is past the window of development to really learn appropriate syntax. So at age 21 years she still poses a question such as, "What we are having for dinner?"

All this time we were alone, negotiating the maze blindly and without a map. Was the smallest problem just the first sign of a bigger problem or just a small problem? What things had we failed to check? What tests had we failed to order? What subspecialists had I failed to ask to see? Or, was she actually doing just fine? There were neither experts to ask nor other families' experiences to draw on. All of Elizabeth's doctors were in the same situation as we were. None had ever treated another child with 18q-. None had the time to go to the medical library and dig out the sparse and outdated information related to this single patient.

As a kid, I was a fan of western television shows and often fantasized about being a pioneer. I dreamt about going west where few others had ever been and seeing sights few others had seen. We built forts from any box or board left unattended and rode to the rescue on our two-wheeled horses. I wished for a life carving out a home from the wilderness where everyday brought new adventure. Well, as they say be careful what you wish for, you might just get it. But what I got was not the kind of pioneering I had envisioned. It is one thing to risk your own life and limb, but risking your child's is an order of magnitude more serious. When you are making decisions about your child's life, health, and future based on obviously bad information, you are definitely taking risks. The risks from inaction could be as devastating as taking the wrong action. These are not the kind of risks any parent should ever be faced with.

Today this need not happen to the affected family or their health care providers. The Chromosome 18 Registry and Research Society exists to improve understanding of the syndromes of chromosome 18. With more than 1800 affected families, the organization is a resource for both physicians and families to find out about things that are common in children with chromosome 18 abnormalities, but rare in the general population. In fact, the families of the Chromosome 18 Registry support the only clinical research center in the world studying these syndromes.

The Chromosome 18 Registry and Research Society is not a unique organization. There are hundreds of similar organizations each dedicated to a specific genetic condition or group of conditions. These organizations come in all sizes, shapes, and colors. Some have blood and tissue banks, some fund substan- 
tial research programs, and some have built awesome political machines. The most amazing thing about virtually all of these organizations is the fact that families of affected individuals started them. They were started without a business plan and without venture capital. They were started with vision and passion; the vision that the lives of others with their condition could be improved and the passion to make it happen.

These organizations not only hold great potential as a resource for families but also can be a huge benefit to physicians. However, making the most of a relationship with a lay advocacy group requires an appreciation of what they actually do. Despite the fact that they are independent, they all provide some aspect of the following three basic functions.

First, they provide a sense of community for the affected individuals. In fact, they often provide the only way for affected individuals with rare conditions to meet another person with the same challenges and perspectives. The value of such friendships cannot be overstated. Differences between people in education, economic status, and political persuasion all evaporate when you actually meet someone who has experienced the same shock of diagnosis and has the same abrupt reorienting of life's priorities. Annual conferences of these organizations should really be called family reunions. These people are members of a family with an enduring bond; it is not just a bond of blood.

This community often includes those who have asked the same question you have now and can tell you what they learned and with whom they consulted for more information. Families built networks through these community connections that not only help find answers but also help disintegrate the feeling of isolation and confusion. If nothing else, you might learn that you are not the only one who is confused about a certain problem.

I sometimes think that the most important and overlooked beneficiaries of these communities are the siblings of the affected individuals. The siblings did not ask for this life eitherdoctor appointments, therapy sessions, and stares from strangers who do not understand. It is often the siblings who will have the longest and lifelong relationship with the affected individual. At the family conferences, siblings get a chance to meet others who do not need explanations and will be perfectly understanding and accepting of their special brother or sister.

Second, most lay advocacy organizations have an educational component to their mission. They educate their members, the medical community, the public, and the policy makers about their condition, needs, and struggles. They do this through conferences, newsletters, and the media. The more rare the condition, the more important their role as a repository of the collected work on that condition. This is because, with rare conditions, the information is less widely and therefore readily available, so the advocacy organization can compile what is known about the condition and be a one-stop resource for the parent and health care community. These organizations are often in a position to know the latest finding before they are actually in press.

Third, they facilitate research. Some organizations actually fund research, but most provide access to a group of potential research participants. Advocacy organizations often fund grants to individual investigators, sponsor scientific meetings, and even fund research centers. They can be a powerful force in setting the research agenda through their influence as a funding source and as an intermediary between competing research groups.

In the same way that conditions vary (from life threatening to mere curiosities) the lay advocacy organizations also vary. This wide degree of difference is due to several factors. First, the type and severity of the condition itself has a profound influence on the focus of the organization. The more life-threatening the condition, usually the more motivated the members of the organization are to learn more, find better treatments, and do the fundraising necessary to help the organization to grow. The primary needs of the affected families are reflected in the primary focus of the organization. These needs could be access to care, funding for research, and a need for grief support or any of a number of issues.

Second, the lay advocacy groups' founders have a great influence on the vision and focus of the organization. Because most groups are started by those directly affected, the skills and background of the founders will mold the direction of the organization. Even those organizations that have grown to have several employees are still principally volunteer driven and have boards of directors who are composed primarily of affected family members. Groups who have leaders with a high level of computer skills are more likely to have fancy websites and web-based donation programs. Those founders with a more artistic bent may have begun a more interpersonal relationship focused group.

Third, the stage of development of the organization will have a great effect on the services offered. Organizations that have been providing services for many years are more likely to have built up a funding base and have paid staff. Because most lay advocacy groups are nonprofit organizations begun by affected families, there are no wealthy investors pouring in money to get it up and running overnight. These organizations are built on sweat equity from kitchen tables, one dollar, one volunteer hour at a time. Many organizations have grown well beyond the kitchen table stage, but most of them started there. Those that are still at this stage should be appreciated for their potential and nurtured through their infancy.

A fourth factor influencing the organization is the incidence of the condition. A condition that affects only a couple of hundred families in this country will have a harder time gathering the recognition and resources needed to grow into a mature organization. More common conditions have an easier time attracting attention, funding, and a celebrity spokesperson.

Despite all these reasons for variability, lay advocacy groups are fundamentally the same. Lay advocacy groups are central repositories for information on a particular condition. They are often a source of literature reviews, fact sheets, and clinical management guidelines. Beyond medical information, they often provide information on educational considerations and day-to-day living issues, such as recipes for special diets and access to assistive technologies, the tried and true wisdom that only comes from "been there done that." 
Possibly most important to health care professionals, lay advocacy groups can also be the link to the leading experts for answers to specific medical questions. Most organizations have close ties to researchers. A call to an organization can quickly yield the contact information for the top experts on a specific aspect of a syndrome. The sheer number of different genetic conditions makes it nearly impossible for anyone to be familiar with the medical issues, much less the latest research findings of each one. Lay advocacy groups provide the link to information about each condition.

The easiest way for a health care provider to keep up with the best information and the latest developments is to create a partnership with the affected individuals or families. Allow and encourage them to become partners in their own health care. Encourage them to find and join the lay advocacy group for their condition. Ask them to keep you informed when they learn something new. These are the individuals who will be frequent patients, because many genetic conditions affect a person throughout his or her life and have a pervasive influence on the person's overall health and lifestyle.

As members of lay advocacy groups, they can make connections to other parents to learn about day-to-day management issues. Through their association with a lay advocacy group, they will know who the experts are and pass this information on to their personal health care providers.

There are many different ways for patients to be involved in a lay advocacy group. Some families thrive on the strength they derive from knowing other families, going to conferences, and working to help the organization. Some families wish to belong, but at a distance. They want to collect information and stay on top of the latest finding, but are not interested in meeting other people or being involved in the organization. Regardless of where on this continuum a family might fit, they all derive benefit from being a member of the organization. Their health care providers can benefit from their involvement as well.

Although there are many organizations, finding the right organization is easy. The Genetic Alliance is an umbrella organization to which most single-disability genetic lay advocacy groups belong. Genetic Lay Advocacy groups can be accessed through the Genetic Alliance web site (www.geneticalliance. org). The site is searchable using the name of the condition or the name of the organization.

Before I started The Chromosome 18 Registry and Research Society 17 years ago, I had no idea all of these organizations existed. It would never have occurred to me to ask any of my daughter's doctors if there was a group for 18q-, because I did not know any condition specific groups even existed. This is a world that was entirely unknown to me. Newly diagnosed individuals and families are unlikely to know that these organizations exist or appreciate the benefits they may bring to their lives. Health care providers can be the bridge to the lay advocacy group, and in return the patients will be able to become partners in their healthcare management. These are great opportunities for building a partnership between physicians and patients and improving medical care and the lives of those affected. 